\title{
MIECTOMÍA SACROCOCCIGEA VENTRAL EN EQUINOS
}

\author{
VENTRAL MIECTOMY SACROCOCCYGEUS IN EQUINE \\ Mastoby Martínez M, ${ }^{*}$ Hernando Martínez C, Alba Sánchez G, Juan Ballut P. \\ Universidad de Córdoba, Facultad de Medicina Veterinaria y Zootecnia, Departamento de Ciencias Pecuarias. \\ A.A 354 Montería, Colombia. ${ }^{*}$ Correspondencia: mastobymvz@ hotmail.com \\ Recibido: Mayo 26 de 2006; Aceptado: Noviembre 30 de 2006
}

\section{RESUMEN}

Se realizó una técnica quirúrgica basada en una incisión pequeña del músculo sacrococcigeo ventral, con el fin de realzar la elegancia del caballo al andar teniendo presente los aspectos bioéticos de la medicina veterinaria. En los animales intervenidos no se presento complicación y se logró el objetivo, por lo que se propone esta técnica quirúrgica para que sea practicada por los veterianrios durante su ejercicio profesional.

Palabras clave: Miectomia, sacrococcigeo, caballos.

\section{ABSTRACT}

A surgical technique based on a small incision of ventral sacrococcygeus muscle was made with the purpose of enhancing the elegance of horse to walk having present bioethical aspects of the veterinary medicine. Intervened animals doesn't present complication and the objective was achieved, for what this surgical technique is proposed to veterinarian for practicing during theirs professional exercise.

Key words: Miectomy sacrococcygeus, horses. 


\section{INTRODUCCIÓN}

A través del tiempo el caballo ha desempeñado un papel importante en las diferentes actividades pecuarias del hombre, quien ha condicionado a estos animales a sus necesidades, dentro de las que se encuentra la alta competencia, que exige un manejo zootécnico y la práctica de algunas cirugías que van orientadas a realzar la elegancia de los animales.

La exposición equina es una de las actividades que no solo trae la satisfacción personal de exponer hermosos ejemplares, sino también el valor comercial, constituyéndose en renglón económico en este medio.

Una de las cirugías estéticas que se practica en los caballos es el picaje de cola, que es realizada por personas sin conocimientos quirúrgicos y que no tienen en cuenta las normas de asepsia, el plano anatómico ni el manejo del dolor intra y postoperatorio. Según Berge y Westhues (1), esta técnica solo emplea la sección de los músculos, los que en algunos casos se presenta fibrosis trayendo como resultado la desviación de la cola y por ende de una cirugía correctiva.

El objetivo del presente trabajo es el de describir una técnica quirúrgica que minimiza las posibilidades de fibrosis y que consiste en realizar una miecto mía parcial, del músculo sacrococcigeo ventral.

\section{MATERIALES Y MÉTODOS}

El trabajo se llevó a cabo en varias fincas del departamento de Córdoba, durante el desarrollo de la asignatura Cirugía perteneciente al currículo de la Facultad de Medicina Veterinaria y Zootecnia de la Universidad de Córdoba. Se intervinieron 20 caballos de diferentes edades, razas y sexo, dedicados a las labores de vaquería.

El protocolo anestésico empleado fue tranquilización con acepromacina a dosis de 1 $\mathrm{mg} / \mathrm{kg}$ de peso vivo y anestesia general con guayacolato de glicerilo al $5 \%$ más tiopental sódico a dosis de $1.1 \mathrm{mg} / \mathrm{kg}$ de peso vivo por vía endovenosa, equivalente a $2.2 \mathrm{ml} / \mathrm{kg}$ de peso vivo. En algunos casos se complemento con infiltración local de lidocaina al $2 \%$ de acuerdo con 10 propuesto por Sumano y O campo (2). La secuencia de los pasos quirúrgicos aparecen en detalle en las figuras 1 a 6 .

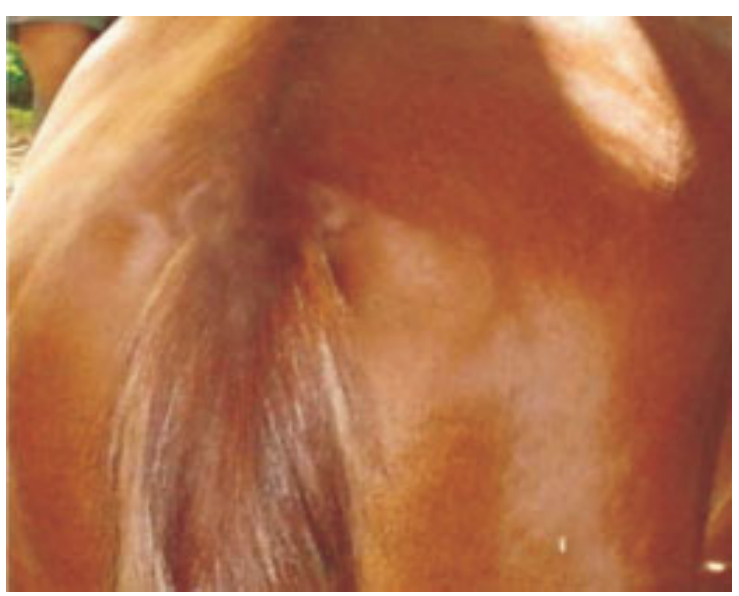

Figura 1: Evaluación caudal y lateral de la cola para determinar su dirección e implantación.

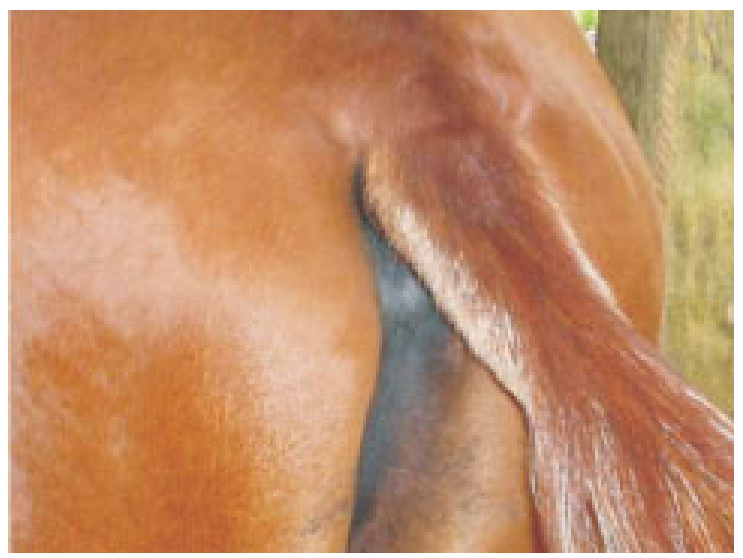

Figura 2: Corte de pelos, rasurado, vendaje de la cola y antisepsia.

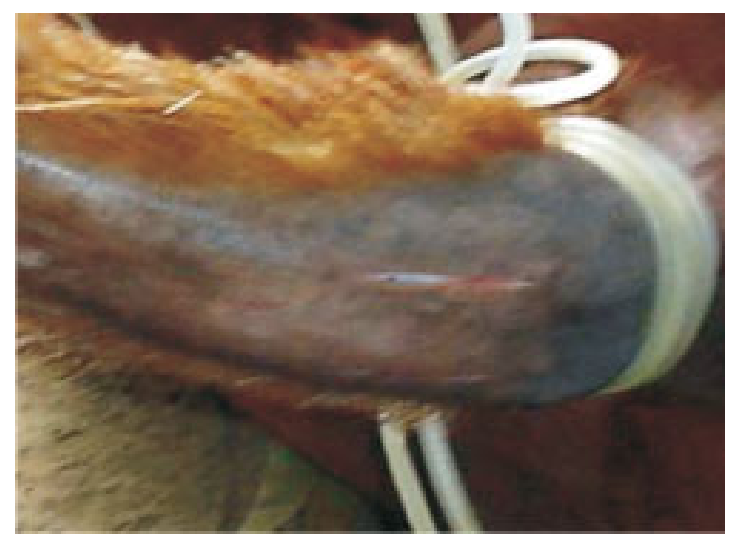

FigurA 3: Colocación de un torniquete con fines hemostáticos. Por debajo del torniquete y a $8 \mathrm{~cm}$ desde la base de la cola (cara ventral); realización de dos incisiones paralelas, exactamente sobre los músculos sacro coccígeos ventrales derecho e izquierdo respectivamente. 


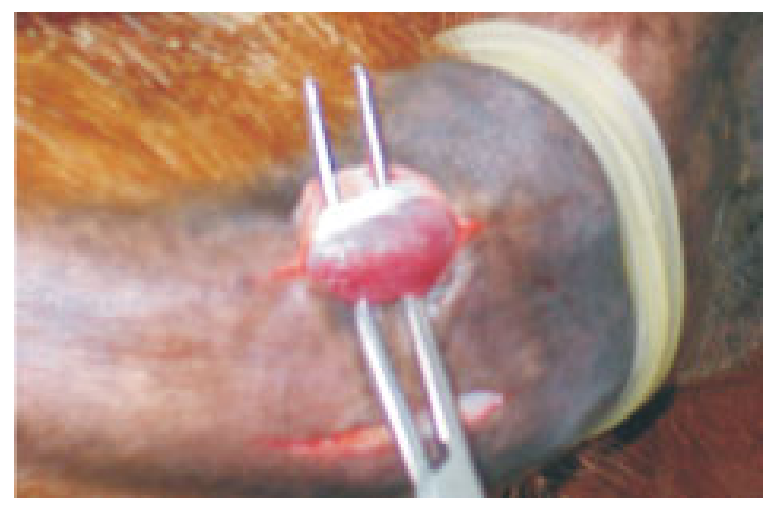

Figura 4: Debridación roma de tejido conectivo y fascia coccígea. Debridación roma de los músculos sacrococcigeo ventrales derecho e izquierdo.

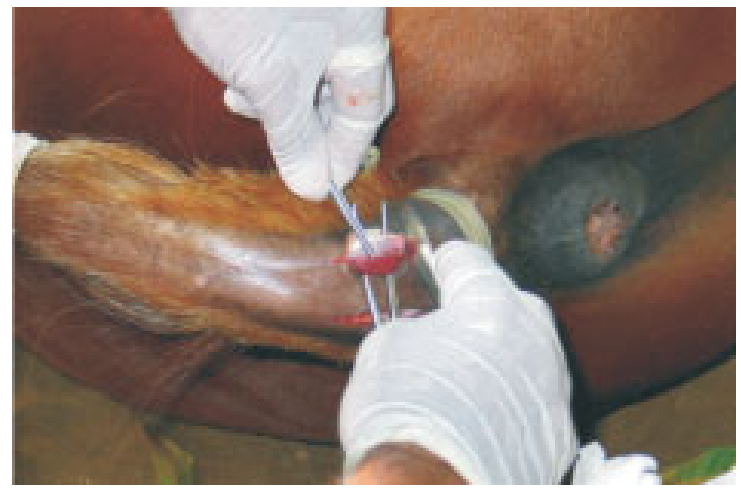

Figura 5: Escisión de 2 centímetros de los músculos sacrococcigeo ventrales derecho e izquierdo.

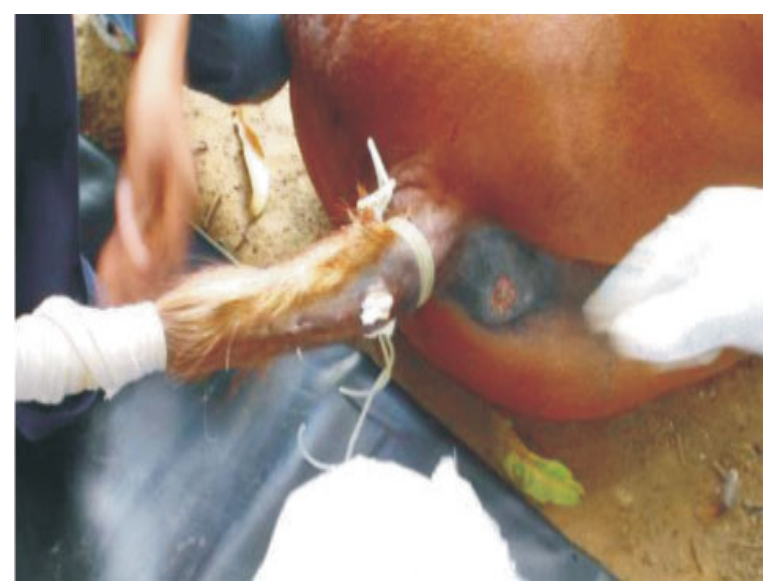

Figura 6: 0 bturación del espacio muerto con to rundas impregnadas con percloruro de hierro para controlar la hemorragia. Estas se retiraron a las 24 horas.

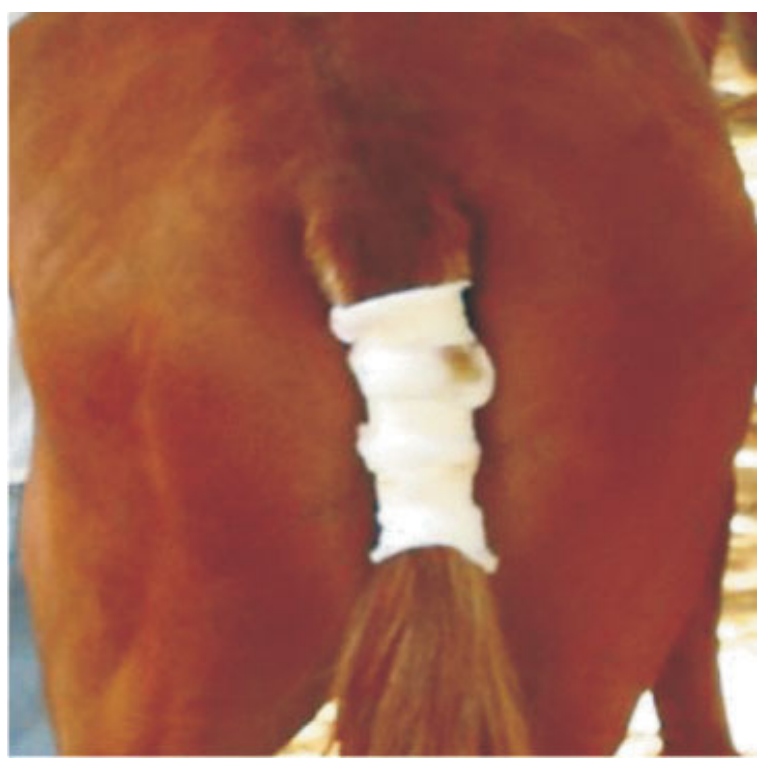

Figura 7: Vendaje final de la cola

Finalmente, se cubrió la cola con gasa (figura $7)$, las cuales se retiraron diariamente. Igualmente, se realizaron las curaciones, según Baxter (3) y Knapp (4), masajes y movimientos suaves de flexión en diferentes sentidos con el objetivo de favorecer el moldeado de la cola.

En algunos casos de ser necesario un segundo abordaje del músculo, y se realizó a unos $5 \mathrm{~cm}$ por debajo de las dos primeras incisiones. La posición del músculo extraído fue menor que en la primera cirugía.

Con esta técnica quirúrgica, se logró dominancia en la acción de los músculos dorsales y laterales de la cola, dando mayor realce y vistosidad de la misma durante el movimiento del animal.

\section{RESULTADOS Y DISCUSIÓN}

La cola en el caballo cumple una función muy importante como mecanismo de defensa contra los ectoparásitos. 0 tra de las funciones es la de servir de equilibrio durante el desplazamiento del animal. Desde el punto de vista zootécnico, la cola representa la vistosidad y elegancia del animal al andar.

De acuerdo con Sisson y G rossman (5); Agüera (6) y Fradsony (7). La cola del equino está constituida por los siguientes planos y 
estructuras anatómicas: externamente por la piel e internamente la fascia coccígea, los músculos sacrococcigeo dorsal, sacrococcigeo lateral, intertransversos, sacrococcigeo ventral, y rectococcigeo. Estos se encuentran en este orden al hacer un corte longitudinal y siguiendo la dirección de las manecillas del reloj (5 - 7). La irrigación e inervación están dadas por los vasos y nervios coccígeos laterales, la arteria y vena coccígea media (5 - 7). Los músculos coccígeos son los encargados de fijar la cola 0 direccionarla dorsal, lateral o ventralmente dependiendo del músculo o grupo de músculos que actúen en el momento (5 - 7).

Tradicionalmente, los criadores de caballo vienen moldeando a los animales de acuerdo a sus gustos y preferencias. El picaje de colas una práctica común en este medio y es practicada por técnicos o personas que no tienen en cuenta las normas de asepsia, planos anatómicos posibles complicaciones intra y post-operatorias. Berge y Westhues (1), describen la técnica como una miotomía subcutánea de la cola. En ella se realiza una disección transversal del músculo. En algunos casos con el paso del tiempo el músculo seccionado se puede adquirir cierta funcionalidad por oposición de tejido fibroso, perdiendo el animal la estética al andar $(2,7)$.

Con la miectomía sacrococcigea ventral se consiguió el objetivo propuesto y una completa cicatrización entre la tercera y cuarta semana. No se observó complicación clínica en los 20 animales estudiados.

Esta cirugía está indicada por su buena implantación de la base de la cola y para que el animal la eleve durante su desplazamiento.

\section{REFERENCIAS}

1. Berge $E$, Westhues $M$. Técnica operatoria veterinaria. 28 a ed. Editorial labor S.A. 1978; p.295-296.

2. Sumano H, O campo L. Farmacología Veterinaria. 1a ed. McG raw Hill. 1988; p.447-461.

3. Baxter M. Manejo de las heridas. En Colan, P; Ian, M; Merrit, A. Medicina y cirugía equina. $4^{\text {a }}$ ed. Vol. II, intermedica editores. 1998; p.1459-1471.

4. Knapp D. Tratamiento de las heridas abiertas. En Sherdin, B. Manual clínico de procedimiento en pequeñas especies Vol. I. 2a ed. McG raw Hill. Interamericana. $2002 ; p .475$.
5. Sisson S, G rossman JD. Anatomía de los animales domésticos. $4^{\text {a }}$ ed. Editorial Salvat Editores, S.A. 1963; p.264.

6. Agüera E, Sandoval J. Anatomía aplicada del caballo. 1 a ed. Hacourt Brace de España S.A. 1999; p.102-103.

7. Fradson R, Supergeon T. Anatomía y fisiología de los animales domésticos. $5^{a}$ ed. Editorial Interamericana. McG raw Hill. 1996; 305-325. 